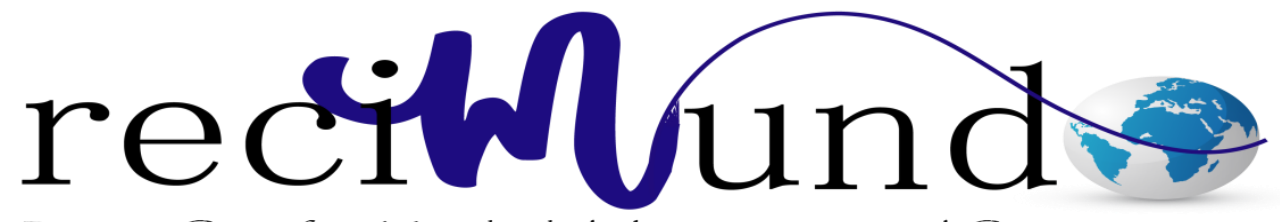

Revista Científica Mundo de la Investigación y el Conocimiento

Cesar Eduardo Chávez Vera ${ }^{a}$; Pedro Napoleón Chara Moreira ${ }^{\text {b; }}$ María Belén Alarcón Valencia ${ }^{c}$; María Milagros Fois Lugo d

Influencia de la fibra de acero en el control de la tenacidad del hormigón simple

Revista Científica Mundo de la Investigación y el Conocimiento. Vol. 2 núm.2, mayo, ISSN: 2588-073X, 2018, pp. 209-235

DOI: 10.26820/recimundo/2.(2).2018.209-235

Editorial Saberes del Conocimiento

Recibido: 05/12/2017

Aceptado: $15 / 03 / 2018$
a. chavezveracesar@gmail.com
b. Universidad de Guayaquil; pedro.charam@ug.edu.ec
c. Universidad de Guayaquil; maria.alarconva@ug.edu.ec
d. 


\section{Influencia de la fibra de acero en el control de la tenacidad del hormigón simple}

Vol. 2, núm. 2., (2018)

Cesar Eduardo Chávez Vera; Pedro Napoleón Chara Moreira; María Belén Alarcón Valencia; María Milagros Fois Lugo

\section{RESUMEN}

El hormigón es un material de uso frecuente en la construcción de viviendas y edificios en el Ecuador, por tal motivo se hace necesario el estudio y la mejora de sus propiedades con el fin de brindar calidad y seguridad en las construcciones.

El hormigón es un material que por sí solo es muy frágil, necesita de aditamentos para la mejora de ciertas propiedades, como es la tenacidad. Para esto son muchos los materiales que se están estudiando, de esta lista sobresalen las fibras de acero, un material muy resistente también pero que hasta el momento solo se lo aplica para obras provisionales como revestimiento de túneles, taludes y pavimentos.

Uso de los objetivos de este informe es brindar a través del conocimiento existente un aporte en cuanto al diseño de hormigón con fibras de acero para la mejora de la tenacidad ante esfuerzos de corte, en base a esto hemos estudiado las características de los agregados, la normativa existente para ensayar varias muestras que nos darán resultados para la evaluación de la tenacidad del hormigón en la ciudad de Guayaquil.

Adicionalmente se estudian otras características del hormigón fresco y de la resistencia a la compresión y flexión (módulo de rotura), las cuales nos darán datos adicionales para mejorar nuestros diseños.

Palabras clave: Hormigón, Fibras, Acero, Tenacidad, Compresión, Módulo de rotura. 


\title{
Influencia de la fibra de acero en el control de la tenacidad del hormigón simple
}

Vol. 2, núm. 2., (2018)

Cesar Eduardo Chávez Vera; Pedro Napoleón Chara Moreira; María Belén Alarcón Valencia; María Milagros Fois Lugo

\begin{abstract}
Concrete is a material often used in the construction of houses and buildings in Ecuador, for this reason the study and improvement of its properties in order to provide quality and safety in construction is necessary.

Concrete is a material that alone is very fragile, adjuncts needs to improve certain properties, such as toughness. To this are many materials being studied, from this list you stands as steel fibers, also a very durable material, but so far only applies to temporary works as lining tunnels, embankments and pavements.
\end{abstract}

Use of the objectives of this report is to provide through the existing knowledge a contribution in the design of concrete with steel fibers to improve the tenacity to shear stresses, on this basis we studied the characteristics of the aggregates, existing regulations to test several samples that will give us results for the evaluation of the toughness of concrete in the city of Guayaquil

In addition other characteristics of fresh concrete and compressive strength and bending (modulus of rupture) they are studied, which will give us additional data to improve our designs.

Key words: Concrete fibers, Steel, Toughness, compression, modulus of rupture. 


\section{Influencia de la fibra de acero en el control de la tenacidad del hormigón simple}

Vol. 2, núm. 2., (2018)

Cesar Eduardo Chávez Vera; Pedro Napoleón Chara Moreira; María Belén Alarcón Valencia; María Milagros Fois Lugo

\section{Introducción.}

Como sabemos, el hormigón simple (HS) es la mezcla ideal de aglomerantes y agregados pétreos, necesaria para fabricar un hormigón de una resistencia determinada, muchos son los estudios que se han realizado a través de ensayos con el objetivo de determinar las características y comportamientos mecánicos de este material con el fin de generalizar su utilización en la industria de la construcción, una de principales conclusiones es la deficiente resistencia de los elementos estructurales fabricados de este material a los esfuerzos cortantes así como un frágil e impactante fisuramiento de las matrices al ser sometidos a cargas y/o solicitaciones.

Siendo algunas las causas que provocan el fisuramiento de un elemento estructural nos centraremos en el análisis de las patologías cuyo origen provienen de la sobre carga de esfuerzos de compresión, flexión y corte, analizando el control de fisuramiento y tenacidad del hormigón endurecido; además de las propiedades del hormigón fresco (segregación, trabajabilidad y consistencia) y la incidencia de estas en la resistencia final de los elementos, que también se puede decir, son producto de un determinado diseño de hormigón y su proceso de fabricación.

Consecuentemente con lo dicho anteriormente, este problema hace que nos planteemos como constructores y sociedad en general, el uso de medidas que mitiguen los efectos no deseados, como es la fisura descontrolada e impactante de los elementos de hormigón cuando fallan, incluso su anulación como elemento estructural, los mismos que pueden conllevar a un colapso parcial o total de las estructuras, poniendo en riesgo la integridad física de los usuarios. Alternativas como el uso de fibras de acero (HRFA) están cada vez entrando con más fuerza en el campo de la construcción, debido a que ha demostrado otorgar apreciables prestaciones al 


\section{Influencia de la fibra de acero en el control de la tenacidad del hormigón simple}

Vol. 2, núm. 2., (2018)

Cesar Eduardo Chávez Vera; Pedro Napoleón Chara Moreira; María Belén Alarcón Valencia; María Milagros Fois Lugo

hormigón simple en el área de la tenacidad, control de fisuras y en menor medida el aumento de la resistencia a la flexión.

El objetivo de la presente investigación es analizar diferentes dosificaciones de fibras de acero en el hormigón simple a partir de la metodología de diseño de hormigones de la ACI para su aplicación en estructuras de hormigo y así incrementar su resistencia a esfuerzos de corte.

\section{Metodología.}

Para la elaboración de este estudio de caso se utilizara el método cuantitativo, el mismo que se caracteriza por basarse en métodos experimentales en complemento con técnicas de estadística y de análisis de resultados.

La utilización de normativa avalada por instituciones nacionales e internacionales, para la realización de ensayos de laboratorio, son las técnicas que predominan en este informe así como la utilización de la teoría actual sobre diseño de hormigones; este método de investigación nos permite manipular y estudiar las características de las variables para finalmente obtener los indicadores que nos permitirán analizar, comprobar resultados que nos permitirán realizar las propuestas finales.

Método de estudio de casos

El estudio de caso, es una estructura investigativa ordenada y directa para conseguir la solución de los problemas planteados a través del conocimiento de las teorías generales, sustantivas y empíricas (objeto y campo de estudio). Metodológicamente se involucra en el 


\section{Influencia de la fibra de acero en el control de la tenacidad del hormigón simple}

Vol. 2, núm. 2., (2018)

Cesar Eduardo Chávez Vera; Pedro Napoleón Chara Moreira; María Belén Alarcón Valencia; María Milagros Fois Lugo

estudio y utilización de instrumentos de recolección de datos y las unidades de análisis para la proposición de variables y obtención de resultados, complementariamente nos este estudio se sustenta en la metodología del marco lógico, estableciendo una identificación precisa del problema, causas, efectos, involucrados, estableciendo una camino crítico hacia el análisis, comprobación y solución del problema.

\section{Premisa}

La incorporación de fibras de acero al hormigón simple incrementa la resistencia a tenacidad del hormigón, mejorando el comportamiento post - fisuramiento de las muestras y extiende el tiempo de fisura o colapso total del hormigón ante esfuerzos de corte y flexión.

Cuadro de categorías, dimensiones, instrumentos y unidades de análisis (CDIU)

\begin{tabular}{|c|c|c|c|}
\hline $\begin{array}{c}\text { CATEGORÍA } \\
\text { S } \\
\end{array}$ & DIMENSIONES & INSTRUMENTOS & UNIDADES DE ANÁLISIS \\
\hline Técnica & $\begin{array}{c}\text { Peso volumétrico } \\
\text { varillado del } \\
\text { agregado grueso } \\
\text { Peso volumétrico } \\
\text { suelto de los } \\
\text { agregados } \\
\text { Densidad } \\
\text { superficialmente } \\
\text { saturada de los } \\
\text { agregados. } \\
\text { Porcentaje de } \\
\text { absorción de los } \\
\text { agregados. } \\
\text { Módulo de finura de } \\
\text { la arena } \\
\text { Relación agua } \\
\text { cemento } \\
\text { Control de } \\
\text { revenimiento } \\
\text { \% de adición de } \\
\text { fibras de acero }\end{array}$ & $\begin{array}{l}\text { - Ficha de ensayos } \\
\text { granulométrico } \\
\text { árido fino } \\
\text { Ficha de ensayos } \\
\text { granulométrico } \\
\text { árido grueso. } \\
\text { - Ficha de ensayos de } \\
\text { viga } \\
\text { - Ficha de ensayos de } \\
\text { cilindros }\end{array}$ & $\begin{array}{l}\text { Muestra de } \\
\text { agregado fino } \\
\text { Muestra de } \\
\text { agregado grueso } \\
\text { Cilindros para } \\
\text { ensayos de } \\
\text { compresión. } \\
\text { Vigas par ensayos } \\
\text { de flexión. }\end{array}$ \\
\hline
\end{tabular}




\section{Influencia de la fibra de acero en el control de la tenacidad del hormigón simple}

Vol. 2, núm. 2., (2018)

Cesar Eduardo Chávez Vera; Pedro Napoleón Chara Moreira; María Belén Alarcón Valencia;

María Milagros Fois Lugo

\begin{tabular}{|c|c|c|c|c|}
\hline Económica & $\begin{array}{c}\text { Peso de fibra de } \\
\text { acero por metro } \\
\text { cubico de hormigón }\end{array}$ & $\begin{array}{c}\text { Proforma de costo } \\
\text { en el mercado. }\end{array}$ & $\begin{array}{c}\text { Fibra de acero } \\
\text { trefilado. }\end{array}$ \\
\hline
\end{tabular}

Descripción de las unidades de análisis

Dentro de las unidades de análisis de este trabajo tenemos principalmente a los agregados de hormigón, sus características físicas nos permiten elaborar un diseño de hormigón adecuado para incrementar la resistencia a tenacidad, estos son:

Agregados Fino y Grueso

El agregado fino es usado en los ensayos son obtenidos a partir de la piedra caliza que se extrae del sitio conocido como cerro azul en la parroquia Chongón del cantón Guayaquil y que es procesada por la empresa Calizas Huayco S.A. Este debe cumplir los requerimientos de las normas NTE-0696 y ASTM-C136 en cuanto la granulometría y se utilizan para la fabricación de productos artificiales resistentes como el hormigón hidráulico (concreto) y el hormigón asfáltico, mediante su mezcla con materiales y aglomerantes tales como: cemento, cal, asfalto, etc.

Cilindros para ensayos de compresión

Son muestras de forma cilíndrica, fabricadas con hormigón hidráulico mediante el uso de moldes metálicos, para su fabricación y control de calidad deben cumplir especificaciones ASTM C-136 ASTM-C31 y ASTM-C192. Previamente, antes de su elaboración se debe realizar un diseño que permita dosificar adecuadamente los agregados utilizados. 


\section{Influencia de la fibra de acero en el control de la tenacidad del hormigón simple}

Vol. 2, núm. 2., (2018)

Cesar Eduardo Chávez Vera; Pedro Napoleón Chara Moreira; María Belén Alarcón Valencia; María Milagros Fois Lugo

Este espécimen como le vamos a llamar tiene la función metodológica de arrojar datos que indiquen la resistencia del mismo ante una carga axial o de compresión según norma NTE1573y ASTM-C39, estos datos deben ser iguales o mayores a los valores de resistencia de diseño.

Vigas par ensayos de flexión

Son muestras de forma prismática, fabricadas con hormigón hidráulico mediante el uso de moldes metálicos, para su fabricación y control de calidad deben cumplir especificaciones según norma ASTM-C31 y ASTM-C192. Previamente, antes de su elaboración se debe realizar un diseño que permita dosificar adecuadamente los agregados utilizados.

Este espécimen como le vamos a llamar tiene la función metodológica de arrojar datos que indiquen la resistencia del mismo apoyado en los extremos y cargado en los tercios de la luz libre, esto según norma NTE-2554 y ASTM-C78, estos datos deben ser iguales o mayores a los valores de resistencia de diseño.

Fibra de acero

Las fibras de acero son pequeños alambres de acero trefilado en frio de $60 \mathrm{~mm}$ longitud y forma alargada que posee ganchos encolados en sus extremos para un mejor rendimiento en el refuerzo del hormigón hidráulico, para lograr su desempeño dentro de la matriz del hormigón deben cumplir con las normativas europeas EN 14889-1 (Dosificación mínima necesaria para cada tipo de fibra).Vienen encoladas en peines para lograr un mezclado rápido, fácil y homogéneo con el hormigón, además de evitar la formación de bolas o grumos de fibras. 


\section{Influencia de la fibra de acero en el control de la tenacidad del hormigón simple}

Vol. 2, núm. 2., (2018)

Cesar Eduardo Chávez Vera; Pedro Napoleón Chara Moreira; María Belén Alarcón Valencia;

María Milagros Fois Lugo

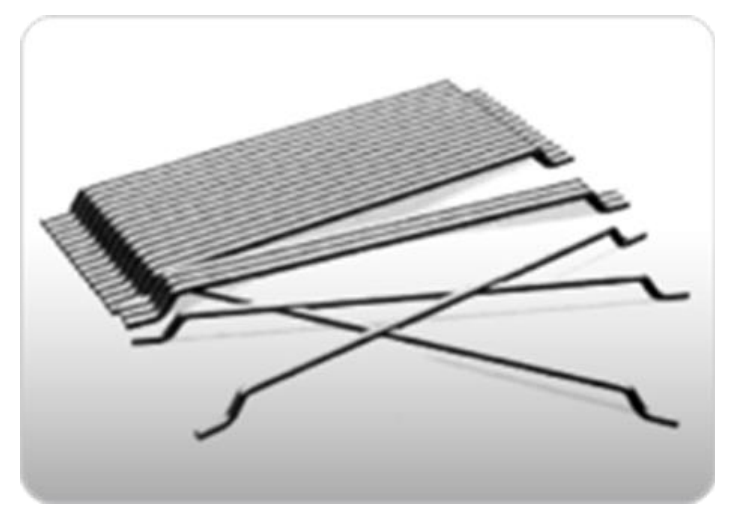

Figura 1.- Presentación de la fibra "Dramix", fuente: Ficha Técnica

Gestión de datos

Considerando la metodología cuantitativa y el carácter rigurosamente técnico de este estudio de caso, se utiliza para la gestión de datos las fichas establecidas por el laboratorio de suelos y hormigones de la Facultad de Ciencias Matemáticas y Físicas de la Universidad de Guayaquil la cual acoge la normativa vigente y que se referencia en el marco teórico en cuanto al análisis granulométrico de los agregados y su posterior caracterización en relación a pesos y densidades.

Por otro lado, refiriéndonos la toma de resultados de los especímenes de hormigón hidráulico, ya sea en cilindros o de viguetas, se ha diseñado la ficha pertinente tomando en consideración los requisitos estipulado a las normas INEN NTE, la cual considera datos como: laboratorio y fecha de ensayo, número de identificación, diámetro de muestra, área de la sección transversal, carga máxima, resistencia a la compresión, tipo de fractura, defectos en cada 


\section{Influencia de la fibra de acero en el control de la tenacidad del hormigón simple}

Vol. 2, núm. 2., (2018)

Cesar Eduardo Chávez Vera; Pedro Napoleón Chara Moreira; María Belén Alarcón Valencia; María Milagros Fois Lugo

espécimen, edad del espécimen, $\mathrm{y}$, densidad, adicionalmente observaciones varias donde se puede señalar responsabilidades sobre los procedimientos de muestreo, transporte y curado de especímenes, además de cualquier variación a los procedimientos señalados en las referidas normas.

Finalmente, todos estos datos son analizados y comparados entre sí, sus características, dimensiones y alternativas de mejora de los resultados y sus respectivos referentes empíricos para establecer las conclusiones correspondientes.

\section{Criterios éticos de la investigación}

La veracidad y confiabilidad de los resultados obtenidos, depende estrictamente del respeto y seguimiento de los procedimientos detallados en cada ensayo, al tratarse de un estudio que se rige en gran medida por bibliografía normativa. 


\section{Influencia de la fibra de acero en el control de la tenacidad del hormigón simple}

Vol. 2, núm. 2., (2018)

Cesar Eduardo Chávez Vera; Pedro Napoleón Chara Moreira; María Belén Alarcón Valencia;

María Milagros Fois Lugo

\section{Resultados.}

Antecedentes de la unidad de análisis

Previamente a la obtención de resultados de tenacidad mediante la rotura de muestras de hormigón hidráulico reforzado con fibras de acero, se tienen que comprobar las características físicas de los agregados siguiendo los parámetros de las NormasNTE-0696 y ASTM-C136, estos parámetros vienen expresados por la siguiente tabla en función del tamaño de los agregados:

\begin{tabular}{|c|c|c|c|c|c|}
\hline \multicolumn{6}{|c|}{ AGREGADO FINO } \\
\hline TAMIZ & $\begin{array}{c}\text { PESO PARCIAL } \\
\text { GRS. }\end{array}$ & \% RETENIDO & $\begin{array}{c}\% \text { RETENIDO } \\
\text { ACUMULADO }\end{array}$ & $\%$ QUE PASA & $\begin{array}{c}\text { ESPECIFICACION } \\
\text { ASTM }\end{array}$ \\
\hline $3 / 8^{\prime \prime}$ & 0 & 0 & 0 & 100 & 100 \\
\hline$N^{\circ} 4$ & 7 & 0,7 & 0,7 & 99,3 & $95-100$ \\
\hline$N^{\circ} 8$ & 147 & 14,7 & 15,4 & 84,6 & $80-100$ \\
\hline$N^{\circ} 16$ & 132 & 13,2 & 28,6 & 71,4 & $50-85$ \\
\hline$N^{\circ} 30$ & 267 & 26,7 & 55,3 & 44,7 & $25-60$ \\
\hline$N^{\circ} 50$ & 179 & 17,9 & 73,2 & 26,8 & $10--30$ \\
\hline $\mathrm{N}^{\circ} 100$ & 244 & 24,4 & 97,6 & 2,4 & $2--10$ \\
\hline FONDO & 24 & 2,4 & 100 & 0 & 0 \\
\hline TOTAL & 1000 & & & & \\
\hline$M F=$ & 2708 & & & & \\
\hline P-V.S. = & $1.379,28$ & $\mathrm{k} / \mathrm{m}^{3}$ & & & \\
\hline Ds.s.s. $=$ & $3.580,00$ & $\mathrm{k} / \mathrm{m}^{3}$ & & & \\
\hline Absorción = & 2,25 & $\%$ & & & \\
\hline
\end{tabular}

Tabla $N^{\circ}$ 1.- Granulometría arena

Revista Científica Mundo de la Investigación y el Conocimiento. 2 (2). pp. 209-235 


\section{Influencia de la fibra de acero en el control de la tenacidad del hormigón simple}

Vol. 2, núm. 2., (2018)

Cesar Eduardo Chávez Vera; Pedro Napoleón Chara Moreira; María Belén Alarcón Valencia; María Milagros Fois Lugo

Una vez realizado el correspondiente tamizado del agregado fino, se determina que cumple con la norma ASTM-C136 para el agregado fino, la cual se encuentra detallada en la columna 6 de la tabla que antecede, por tal motivo no se requiere de ninguna corrección del agregado y es apto para la fabricación de hormigones hidráulicos.

\begin{tabular}{|c|c|c|c|c|c|}
\hline \multicolumn{6}{|c|}{ AGREGADO GRUESO (Tamaño máximo 3/4") } \\
\hline TAMIZ & $\begin{array}{c}\text { PESO PARCIAL } \\
\text { GRS. }\end{array}$ & \% RETENIDO & $\begin{array}{c}\text { \% RETENIDO } \\
\text { ACUMULADO }\end{array}$ & $\%$ QUE PASA & $\begin{array}{c}\text { ESPECIFICACION } \\
\text { ASTM } 3 / 4 "\end{array}$ \\
\hline \multicolumn{6}{|l|}{$21 / 2^{\prime \prime}$} \\
\hline \multicolumn{6}{|l|}{$2 "$} \\
\hline \multicolumn{6}{|l|}{$11 / 2^{\prime \prime}$} \\
\hline $1^{\prime \prime}$ & & & & & 100 \\
\hline 3/4" & 241 & 4,70 & 4,70 & 95,30 & $90--100$ \\
\hline $1 / 2^{\prime \prime}$ & & 0,00 & 4,70 & & \\
\hline $3 / 8^{\prime \prime}$ & 3966 & 77,40 & 82,10 & 17,90 & $20--5$ \\
\hline$N^{\circ} 4$ & 666,2 & 13,00 & 95,11 & 4,89 & $0--10$ \\
\hline$N^{\circ} 8$ & 184,5 & 3,60 & 98,71 & 1,29 & $0--5$ \\
\hline FONDO & 66,3 & 1,29 & 100,00 & 0,00 & \\
\hline TOTAL & 5124 & 100,00 & & & \\
\hline & & & & & \\
\hline P.V.V. = & $1.540,00$ & $\mathrm{k} / \mathrm{m}^{3}$ & & & \\
\hline P.V.S. = & $1.410,00$ & $\mathrm{k} / \mathrm{m}^{3}$ & & & \\
\hline D.s.s.s. $=$ & $2.480,00$ & $\mathrm{k} / \mathrm{m}^{3}$ & & & \\
\hline Absorción = & 2,93 & $\%$ & & & \\
\hline
\end{tabular}

Tabla $N^{\circ}$ 2.- Granulometría de la grava

Se realiza también el análisis granulométrico del agregado grueso según normaASTMC136 para el agregado grueso de tamaño máximo 3/4", la cual se encuentra detallada en la columna 6 de la tabla que antecede, por tal motivo no se requiere de ninguna corrección del agregado y es apto para la fabricación de hormigones hidráulicos.

Por otro lado, se realiza el correspondiente diseño de hormigón hidráulico para la elaboración de las muestras mediante el método ACI, el cual parte de las características físicas de 


\section{Influencia de la fibra de acero en el control de la tenacidad del hormigón simple}

Vol. 2, núm. 2., (2018)

Cesar Eduardo Chávez Vera; Pedro Napoleón Chara Moreira; María Belén Alarcón Valencia; María Milagros Fois Lugo

los agregados y de requerimientos de resistencia y trabajabilidad del hormigón; es así que se escoge realizar un diseño de hormigón estructural que para nuestro estudio será de $210 \mathrm{Kg} / \mathrm{cm} 2$ con un revenimiento de 15 a $20 \mathrm{~cm}$., a este hormigón base le añadiremos dos distintas dosificaciones de fibra de acero para su posterior análisis. 


\section{Influencia de la fibra de acero en el control de la tenacidad del hormigón simple}

Vol. 2, núm. 2., (2018)

Cesar Eduardo Chávez Vera; Pedro Napoleón Chara Moreira; María Belén Alarcón Valencia; María Milagros Fois Lugo

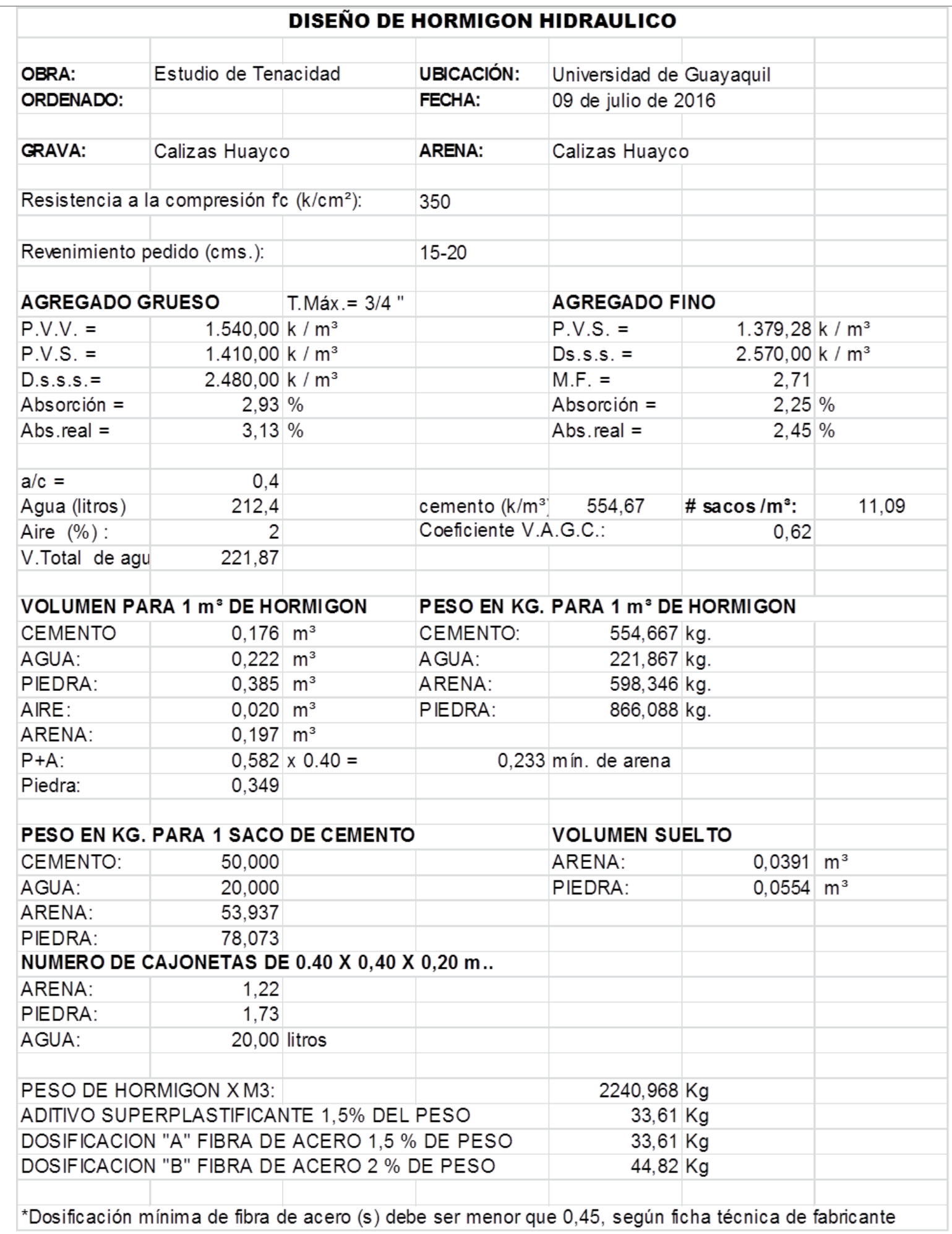

Revista Científica Mundo de la Investigación y el Conocimiento. 2 (2). pp. 209-235 


\section{Influencia de la fibra de acero en el control de la tenacidad del hormigón simple}

Vol. 2, núm. 2., (2018)

Cesar Eduardo Chávez Vera; Pedro Napoleón Chara Moreira; María Belén Alarcón Valencia;

María Milagros Fois Lugo

\section{Tabla $N^{\circ}$ 3.- Diseño de hormigón}

Para determinar la cantidad de hormigón a preparar, se enumera los ensayos a realizarse y se cuantifica el número de especímenes de acuerdo a lo que la norma requiere,(Terreros de Varela, 2006) considera: "Para cada ensayo de resistencia deberá tomarse por lo menos 2 especímenes de ensayo su resultado será el promedio de los 2 especímenes ensayados a la humedad especificada" (p.171), es así que:

\section{Cuadro $N^{\circ}$ 1.- Análisis de muestras}

\begin{tabular}{|c|c|c|c|}
\hline NORMA & $\begin{array}{c}\text { DESCRIPCIÓN DE } \\
\text { ENSAYO }\end{array}$ & TIPO DE MUESTRA & $\begin{array}{c}\text { CANTIDAD DE } \\
\text { MUESTRAS }\end{array}$ \\
\hline $\begin{array}{c}\text { NTE INEN-1573 } \\
\text { ASTM C-39 }\end{array}$ & $\begin{array}{c}\text { Resistencia a la } \\
\text { compresión }\end{array}$ & $\begin{array}{c}\text { Cilindros de hormigón } \\
\text { hidráulico }\end{array}$ & $\begin{array}{c}12 \text { (2 roturas por } \\
\text { muestra a 7y 28 días) }\end{array}$ \\
\hline $\begin{array}{c}\text { NTE INEN-2554 } \\
\text { ASTM C-78 }\end{array}$ & $\begin{array}{c}\text { Resistencia a la flexión } \\
\text { (carga en los tercios) }\end{array}$ & $\begin{array}{c}\text { Vigueta de hormigón } \\
\text { hidráulico }\end{array}$ & $\begin{array}{c}12 \text { (2 roturas por } \\
\text { muestra a } 7 \text { y 28 días) }\end{array}$ \\
\hline
\end{tabular}

Presentación de los resultados.

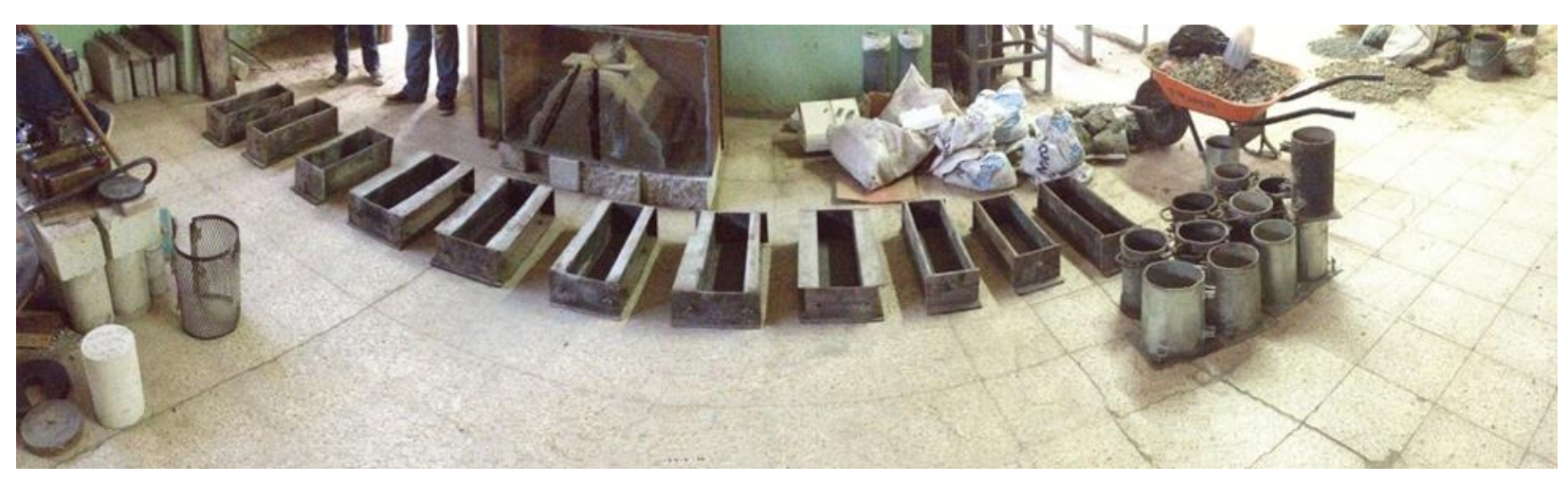

Figura $N^{\circ}$ 1.- Moldes utilizados para muestras 


\section{Influencia de la fibra de acero en el control de la tenacidad del hormigón simple}

Vol. 2, núm. 2., (2018)

Cesar Eduardo Chávez Vera; Pedro Napoleón Chara Moreira; María Belén Alarcón Valencia; María Milagros Fois Lugo

Durante la fabricación de los especímenes se siguieron los procedimientos regulares para la correcta colocación del hormigón de los moldes, la mezcla de los agregados se la realizó por medios mecánicos con el uso de mezcladora. Para la colocación en los moldes, se trabajó en capas de $10 \mathrm{~cm}$ en cilindros, a continuación se varilló la mezcla y golpeó exteriormente los moldes para lograr una óptima compactación.

\section{Revenimiento del hormigón hidráulico.}

Luego del mezclado del hormigón, inmediatamente se mide la consistencia de la mezcla mediante el uso del cono de abrams, a continuación los resultados:
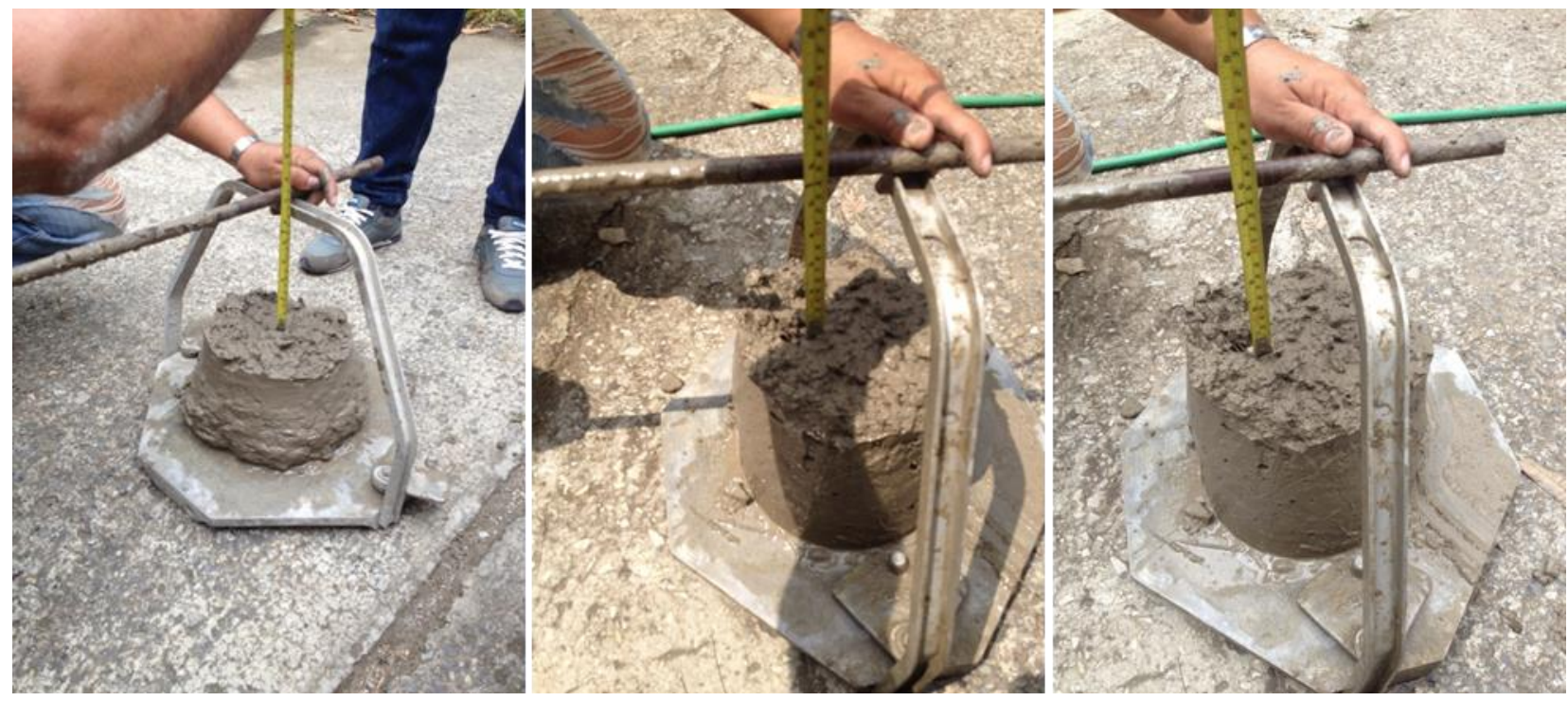

Figura $N^{\circ}$ 2.- Análisis de consistencia 


\section{Influencia de la fibra de acero en el control de la tenacidad del hormigón simple}

Vol. 2, núm. 2., (2018)

Cesar Eduardo Chávez Vera; Pedro Napoleón Chara Moreira; María Belén Alarcón Valencia; María Milagros Fois Lugo

\section{Cuadro $N^{\circ}$ 2.- Resumen de consistencias}

\section{Laboratorio donde se realizó el ensayo: Ing. Dr. Arnaldo Ruffilli}

Ubicación: Universidad de Guayaquil, Provincia del Guayas

\begin{tabular}{|c|c|c|}
\hline Nombre de Muestra & Revenimiento de diseño $(\mathrm{cm})$. & Revenimiento obtenido (cm.) \\
\hline HS (Hormigón base) & $15-20$ & 17 \\
\hline HRFA “A" & $15-20$ & 15 \\
\hline HRFA "B" & $15-20$ & 14 \\
\hline
\end{tabular}

El revenimiento del hormigón base está dentro de los parámetros de diseño, no así en hormigón con fibras, este pierde consistencia debido a la incorporación de las fibras de acero en un $15 \%$.

\section{Ensayos de compresión}

Se fabricaron cilindros para analizar el comportamiento de estos a la resistencia a compresión, las roturas se hicieron a los 7 y 28 días de curado. La dimensión de los especímenes es de $15 \mathrm{~cm}$ de diámetro y $30 \mathrm{~cm}$ de altura y fueron ensayados en el Laboratorio Dr. Arnaldo Ruffilli de la Universidad Guayaquil. Se fabricaron tres tipos de hormigón cuya diferencia es el contenido de fibra, el diseño y la dosificación de los agregados así como aditivos se mantuvo en todos los especímenes. 


\section{Influencia de la fibra de acero en el control de la tenacidad del hormigón simple}

Vol. 2, núm. 2., (2018)

Cesar Eduardo Chávez Vera; Pedro Napoleón Chara Moreira; María Belén Alarcón Valencia; María Milagros Fois Lugo

Primera ronda de ensayos

\begin{tabular}{|c|c|c|c|c|c|}
\hline \multicolumn{6}{|c|}{ Laboratorio donde se realizó el ensayo: Ing. Dr. Arnaldo Ruffilli } \\
\hline \multicolumn{6}{|c|}{ Ubicación: Universidad de Guayaquil, Provincia del Guayas } \\
\hline $\begin{array}{ll}\text { Nombre } & \text { de } \\
\text { Muestra } & \end{array}$ & $\begin{array}{l}\text { Fecha de } \\
\text { fabricación }\end{array}$ & \begin{tabular}{|l|}
$\begin{array}{l}\text { Fecha } \\
\text { rotura }\end{array}$ \\
\end{tabular} & $\begin{array}{l}\text { Edad de la } \\
\text { muestra }\end{array}$ & $\begin{array}{l}\text { Carga } \\
\text { máxima }\end{array}$ & $\begin{array}{l}\text { F'c } \\
(\mathrm{Kg} / \mathrm{cm} 2)\end{array}$ \\
\hline $\begin{array}{ll}\text { HS } & \text { (Hormigón } \\
\text { base) } & \\
\end{array}$ & $09 / 07 / 2016$ & $16 / 07 / 2016$ & 7 & 22500 & 127,32 \\
\hline HRFA “A” & 09/07/2016 & $16 / 07 / 2016$ & 7 & 21000 & 118,83 \\
\hline HRFA "B" & $09 / 07 / 2016$ & $16 / 07 / 2016$ & 7 & 20000 & 113,17 \\
\hline
\end{tabular}

Segunda ronda de ensayos

\begin{tabular}{|l|l|l|l|l|l|}
\hline \multicolumn{6}{|l|}{ Laboratorio donde se realizó el ensayo: Ing. Dr. Arnaldo Ruffilli } \\
\hline Ubicación: Universidad de Guayaquil, Provincia del Guayas \\
\hline $\begin{array}{l}\text { Nombre de } \\
\text { Muestra }\end{array}$ & $\begin{array}{l}\text { Fecha de } \\
\text { fabricación }\end{array}$ & $\begin{array}{l}\text { Fecha de } \\
\text { rotura }\end{array}$ & $\begin{array}{l}\text { Edad de la } \\
\text { muestra }\end{array}$ & $\begin{array}{l}\text { Carga } \\
\text { máxima }\end{array}$ & $\begin{array}{l}\text { F'c } \\
\text { (Kg/cm2) }\end{array}$ \\
\hline HS (Hormigón base) & $09 / 07 / 2016$ & $16 / 07 / 2016$ & 7 & 23000 & 130,15 \\
\hline HRFA “A” & $09 / 07 / 2016$ & $16 / 07 / 2016$ & 7 & 21000 & 118,83 \\
\hline HRFA “B” & $09 / 07 / 2016$ & $16 / 07 / 2016$ & 7 & 19000 & 107,51 \\
\hline
\end{tabular}

Resumen de ensayo a compresión a los 7 días

\begin{tabular}{|l|c|r|r|r|}
\hline $\begin{array}{c}\text { Nombre de } \\
\text { Muestra }\end{array}$ & $\begin{array}{c}\text { Edad de la } \\
\text { muestra }\end{array}$ & $\begin{array}{c}\text { F'c } \\
\text { obtenida } \\
\text { (Kg/cm2) }\end{array}$ & $\begin{array}{c}\text { F'c de } \\
\text { diseño } \\
\text { (Kg/cm2) }\end{array}$ & (\%) \\
\hline HS (Hormigón base) & 7 días & 128,735 & 210 & 61 \\
\hline HRFA "A" & 7 días & 118,83 & 210 & 57 \\
\hline HRFA "B" & 7 días & 110,34 & 210 & 53 \\
\hline
\end{tabular}

Revista Científica Mundo de la Investigación y el Conocimiento. 2 (2). pp. 209-235 


\section{Influencia de la fibra de acero en el control de la tenacidad del hormigón simple}

Vol. 2, núm. 2., (2018)

Cesar Eduardo Chávez Vera; Pedro Napoleón Chara Moreira; María Belén Alarcón Valencia; María Milagros Fois Lugo

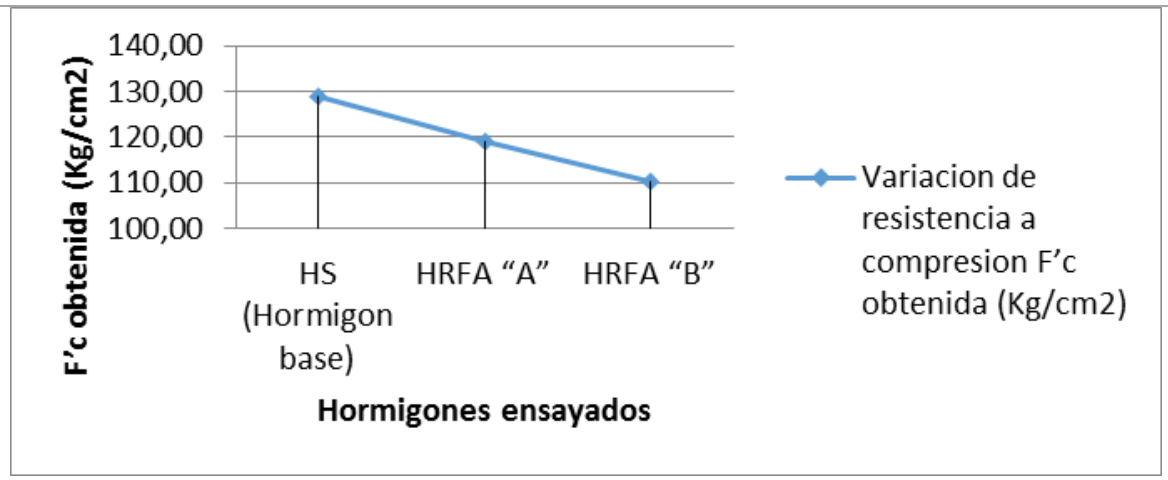

Tercera ronda de ensayos

\begin{tabular}{|l|c|l|l|l|l|}
\hline \multicolumn{6}{|l|}{ Laboratorio donde se realizó el ensayo: Ing. Dr. Arnaldo Ruffilli } \\
\hline Ubicación: Universidad de Guayaquil, Provincia del Guayas \\
\hline $\begin{array}{l}\text { Nombre de } \\
\text { Muestra }\end{array}$ & $\begin{array}{l}\text { Fecha de } \\
\text { fabricación }\end{array}$ & $\begin{array}{l}\text { Fecha de } \\
\text { rotura }\end{array}$ & $\begin{array}{l}\text { Edad de la } \\
\text { muestra }\end{array}$ & $\begin{array}{l}\text { Carga } \\
\text { máxima }\end{array}$ & $\begin{array}{l}\text { F'c } \\
\text { (Kg/cm2) }\end{array}$ \\
\hline HS (Hormigón base) & $09 / 07 / 2016$ & $06 / 08 / 2016$ & 28 & 35000 & 198,05 \\
\hline HRFA “A" & $09 / 07 / 2016$ & $06 / 08 / 2016$ & 28 & 30000 & 169,76 \\
\hline HRFA "B" & $09 / 07 / 2016$ & $06 / 08 / 2016$ & 28 & 36000 & 203,71 \\
\hline
\end{tabular}

Cuarta ronda de ensayos

\begin{tabular}{|l|l|l|l|l|r|}
\hline \multicolumn{6}{|l|}{ Laboratorio donde se realizó el ensayo: Ing. Dr. Arnaldo Ruffilli } \\
\hline Ubicación: Universidad de Guayaquil, Provincia del Guayas \\
\hline $\begin{array}{l}\text { Nombre de } \\
\text { Muestra }\end{array}$ & $\begin{array}{l}\text { Fecha de } \\
\text { fabricación }\end{array}$ & $\begin{array}{l}\text { Fecha de } \\
\text { rotura }\end{array}$ & $\begin{array}{l}\text { Edad de la } \\
\text { muestra }\end{array}$ & $\begin{array}{l}\text { Carga } \\
\text { máxima }\end{array}$ & $\begin{array}{l}\text { F'c } \\
\text { (Kg/cm2) }\end{array}$ \\
\hline HS (Hormigón base) & $09 / 07 / 2016$ & $06 / 08 / 2016$ & 28 & 37000 & 209,37 \\
\hline HRFA “A" & $09 / 07 / 2016$ & $06 / 08 / 2016$ & 28 & 35000 & 198,05 \\
\hline HRFA "B" & $09 / 07 / 2016$ & $06 / 08 / 2016$ & 28 & 34000 & 192,39 \\
\hline
\end{tabular}

Resumen de ensayo a compresión a los 7 días

\begin{tabular}{|l|c|r|r|r|}
\hline $\begin{array}{c}\text { Nombre de } \\
\text { Muestra }\end{array}$ & $\begin{array}{c}\text { Edad de la } \\
\text { muestra }\end{array}$ & $\begin{array}{c}\text { F'c } \\
\text { obtenida } \\
\text { (Kg/cm2) }\end{array}$ & $\begin{array}{c}\text { F'c de } \\
\text { diseño } \\
\text { (Kg/cm2) }\end{array}$ & (\%) \\
\hline HS (Hormigón base) & 28 días & 203,71 & 210 & 97 \\
\hline HRFA “A" & 28 días & 183,91 & 210 & 88 \\
\hline HRFA "B" & 28 días & 198,05 & 210 & 94 \\
\hline
\end{tabular}

Revista Científica Mundo de la Investigación y el Conocimiento. 2 (2). pp. 209-235 


\section{Influencia de la fibra de acero en el control de la tenacidad del hormigón simple}

Vol. 2, núm. 2., (2018)

Cesar Eduardo Chávez Vera; Pedro Napoleón Chara Moreira; María Belén Alarcón Valencia; María Milagros Fois Lugo

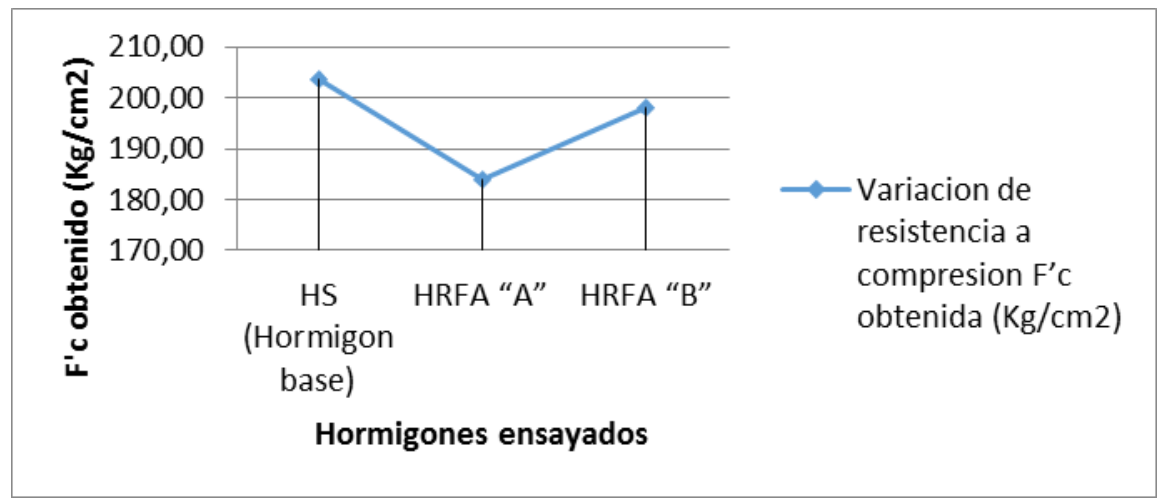

\section{Ensayos de flexión y curva de tenacidad}

Obtención del Módulo de Rotura (Ensayo de Flexión)

\begin{tabular}{|c|c|c|c|c|c|}
\hline \multicolumn{6}{|c|}{ Laboratorio donde se realizó el ensayo: Ing. Dr. Arnaldo Ruffilli } \\
\hline \multicolumn{6}{|c|}{ Ubicación: Universidad de Guayaquil, Provincia del Guayas } \\
\hline $\begin{array}{c}\begin{array}{c}\text { Nombre de } \\
\text { Muestra }\end{array} \\
\end{array}$ & $\begin{array}{c}\text { Fecha de } \\
\text { fabricación }\end{array}$ & $\begin{array}{c}\text { Fecha de } \\
\text { rotura }\end{array}$ & $\begin{array}{c}\text { Edad de la } \\
\text { muestra }\end{array}$ & $\begin{array}{c}\text { Carga } \\
\text { máxima } \\
\end{array}$ & $\begin{array}{c}\text { MR } \\
(\mathrm{Kg} / \mathrm{cm} 2) \\
\end{array}$ \\
\hline HS (Hormigón base) & $09 / 07 / 2016$ & 06/08/2016 & 28 & 1560 & 20,80 \\
\hline HRFA “A” & 09/07/2016 & 06/08/2016 & 28 & 2700 & 36,00 \\
\hline HRFA “B” & 09/07/2016 & 06/08/2016 & 28 & 2780 & 37,07 \\
\hline
\end{tabular}

\begin{tabular}{|c|c|c|c|c|c|}
\hline \multicolumn{6}{|c|}{ Laboratorio donde se realizó el ensayo: Ing. Dr. Arnaldo Ruffilli } \\
\hline \multicolumn{6}{|c|}{ Ubicación: Universidad de Guayaquil, Provincia del Guayas } \\
\hline $\begin{array}{l}\text { Nombre de } \\
\text { Muestra }\end{array}$ & $\begin{array}{c}\text { Fecha de } \\
\text { fabricación }\end{array}$ & $\begin{array}{l}\text { Fecha de } \\
\text { rotura }\end{array}$ & $\begin{array}{l}\text { Edad de la } \\
\text { muestra }\end{array}$ & $\begin{array}{c}\text { Carga } \\
\text { máxima }\end{array}$ & $\begin{array}{c}\text { MR } \\
(\mathrm{Kg} / \mathrm{cm} 2)\end{array}$ \\
\hline HS (Hormigón base) & $09 / 07 / 2016$ & $06 / 08 / 2016$ & 28 & 1560 & 20,80 \\
\hline HRFA “A” & $09 / 07 / 2016$ & $06 / 08 / 2016$ & 28 & 2640 & 35,20 \\
\hline HRFA "B" & $09 / 07 / 2016$ & $06 / 08 / 2016$ & 28 & 2700 & 36,00 \\
\hline
\end{tabular}

\begin{tabular}{|c|c|c|c|c|}
\hline $\begin{array}{c}\text { Nombre de } \\
\text { Muestra }\end{array}$ & $\begin{array}{c}\text { Edad de la } \\
\text { muestra }\end{array}$ & $\begin{array}{c}\text { MR } \\
\text { obtenido } \\
(\mathrm{Kg} / \mathrm{cm} 2)\end{array}$ & $\begin{array}{c}\text { MR de } \\
\text { diseño } \\
(\mathrm{Kg} / \mathrm{cm} 2)\end{array}$ & $(\boldsymbol{\%})$ \\
\hline
\end{tabular}




\section{Influencia de la fibra de acero en el control de la tenacidad del hormigón simple}

Vol. 2, núm. 2., (2018)

Cesar Eduardo Chávez Vera; Pedro Napoleón Chara Moreira; María Belén Alarcón Valencia;

María Milagros Fois Lugo

\begin{tabular}{|l|l|r|r|r|}
\hline HS (Hormigón base) & 28 días & 20,80 & 21 & 99 \\
\hline HRFA “A” & 28 días & 35,60 & 21 & 170 \\
\hline HRFA “B” & 28 días & 36,53 & 21 & 174 \\
\hline
\end{tabular}

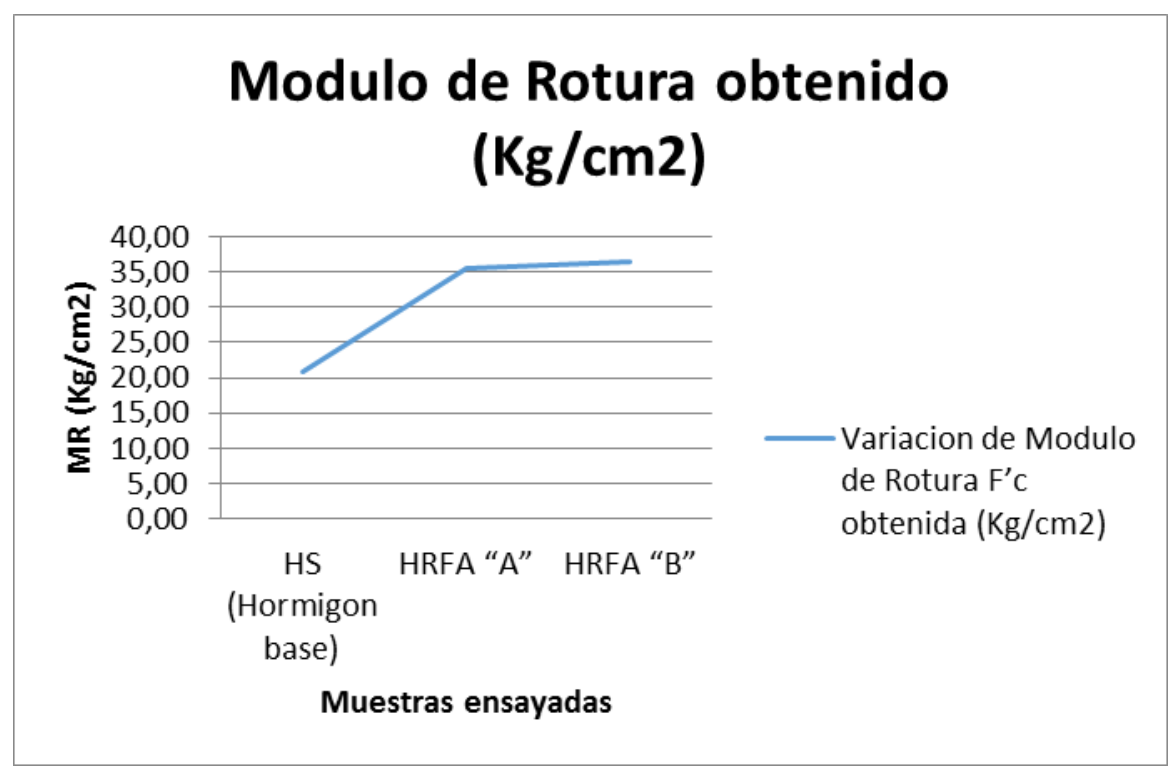

Obtención de la curva Esfuerzo deformación (Ensayo de Flexión) 


\section{Influencia de la fibra de acero en el control de la tenacidad del hormigón}

simple

Vol. 2, núm. 2., (2018)

Cesar Eduardo Chávez Vera; Pedro Napoleón Chara Moreira; María Belén Alarcón Valencia; María Milagros Fois Lugo

\begin{tabular}{|c|c|c|c|}
\hline \multicolumn{4}{|c|}{ DATOS ESFUERZO-DEFORMACION HS } \\
\hline Laboratorio: & Ing. Dr. Arnaldo Ruffilli & & \\
\hline Ubicación: & \multicolumn{2}{|c|}{ Universidad de Gua ya quil } & \\
\hline Tamaño de mues tra: & $50 \times 15 \times 15 \mathrm{cms}$ & Distancia entre apoyos: & $45 \mathrm{cms}$. \\
\hline Fecha mues tra.: & \multirow{2}{*}{\multicolumn{2}{|c|}{\begin{tabular}{l|l}
$09 / 07 / 2016$ & Fecha rotura: \\
$0,54 \mathrm{~mm}$. Según norma UNE 83510
\end{tabular}}} & $06 / 08 / 2016$ \\
\hline Flecha final: & & & \\
\hline \multicolumn{2}{|c|}{ CARGA (N) } & \multicolumn{2}{|c|}{ DEFORMACIÓN (mm.): } \\
\hline \multicolumn{2}{|c|}{0} & \multicolumn{2}{|c|}{0,000} \\
\hline \multicolumn{2}{|c|}{392} & \multicolumn{2}{|c|}{0,005} \\
\hline \multicolumn{2}{|c|}{588} & \multicolumn{2}{|c|}{0,010} \\
\hline \multicolumn{2}{|c|}{784} & \multicolumn{2}{|c|}{0,015} \\
\hline \multicolumn{2}{|c|}{1176} & \multicolumn{2}{|c|}{0,020} \\
\hline \multicolumn{2}{|c|}{1372} & \multicolumn{2}{|c|}{0,025} \\
\hline \multicolumn{2}{|c|}{3234} & \multicolumn{2}{|c|}{0,030} \\
\hline \multicolumn{2}{|c|}{4018} & \multicolumn{2}{|c|}{0,035} \\
\hline \multicolumn{2}{|c|}{4802} & \multicolumn{2}{|c|}{0,040} \\
\hline \multicolumn{2}{|c|}{5586} & \multicolumn{2}{|c|}{0,045} \\
\hline \multicolumn{2}{|c|}{6860} & \multicolumn{2}{|c|}{0,050} \\
\hline \multicolumn{2}{|c|}{8232} & \multicolumn{2}{|c|}{0,055} \\
\hline \multicolumn{2}{|c|}{10094} & \multicolumn{2}{|c|}{0,060} \\
\hline \multicolumn{2}{|c|}{12250} & \multicolumn{2}{|c|}{0,065} \\
\hline \multicolumn{2}{|c|}{14700} & \multicolumn{2}{|c|}{0,070} \\
\hline & 150 & & 075 \\
\hline & 208 & & 080 \\
\hline & 148 & & 085 \\
\hline
\end{tabular}

\section{Curva Esfuerzo Deformación HRFA}

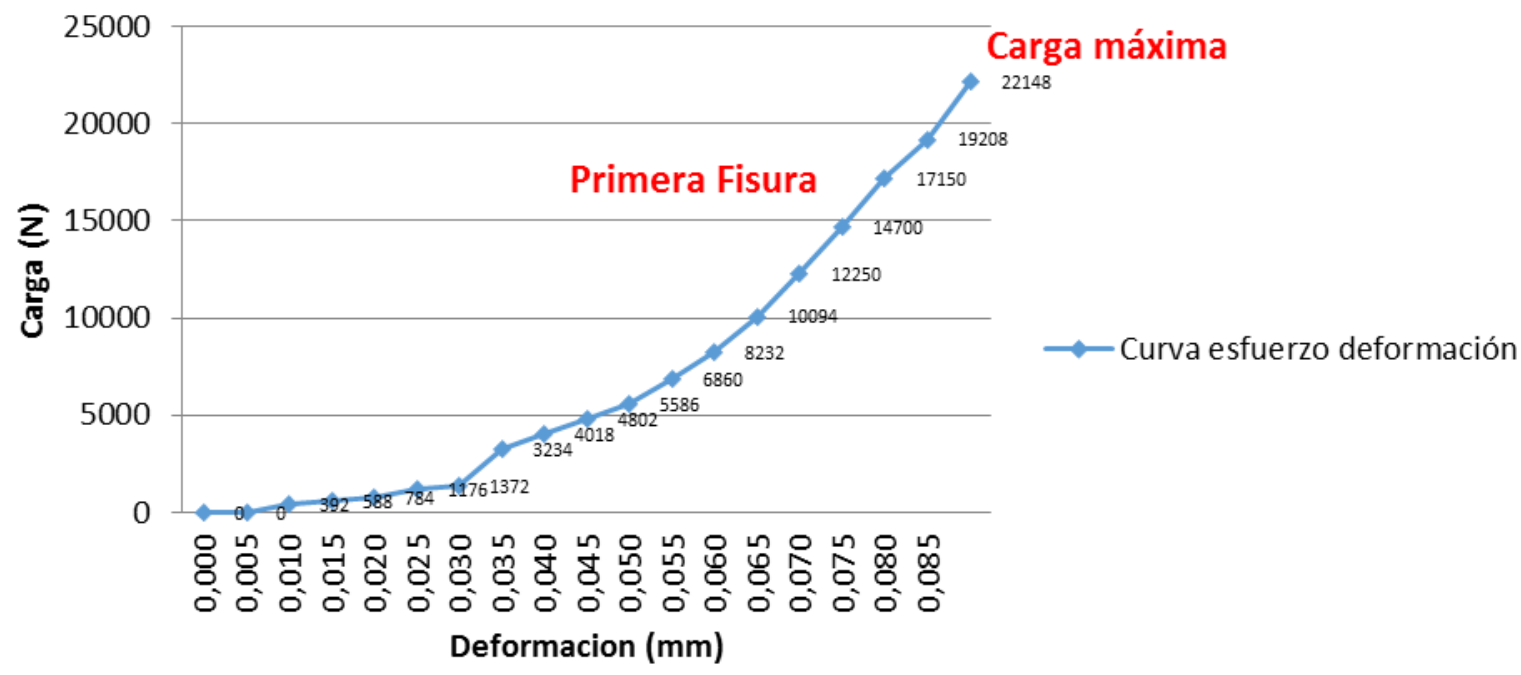




\section{Influencia de la fibra de acero en el control de la tenacidad del hormigón simple}

Vol. 2, núm. 2., (2018)

Cesar Eduardo Chávez Vera; Pedro Napoleón Chara Moreira; María Belén Alarcón Valencia;

María Milagros Fois Lugo

\section{Curva esfuerzo deformación Hormigón}

\begin{tabular}{|c|c|c|c|}
\hline \multicolumn{4}{|c|}{ DATOS ESFUERZO-DEFORMACION HRFA } \\
\hline Laboratorio: & Ing. Dr. Arnaldo R & & \\
\hline Ubicación: & \multicolumn{2}{|c|}{ Universidad de Guayaquil } & \\
\hline Tamaño de mues tra: & $50 \times 15 \times 15 \mathrm{cms}$. & Distancia entre a poyos: & $45 \mathrm{cms}$. \\
\hline Fecha mues tra.: & $09 / 07 / 2016$ & Fecha rotura: & $06 / 08 / 2016$ \\
\hline Flecha final: & \multicolumn{2}{|c|}{ 1,92 mm. Según norma UNE 83510} & \\
\hline \multicolumn{2}{|c|}{ CARGA (N) } & \multicolumn{2}{|c|}{ DEFORMACIÓN (mm.): } \\
\hline \multicolumn{2}{|c|}{0} & \multicolumn{2}{|c|}{0,000} \\
\hline \multicolumn{2}{|c|}{392} & \multicolumn{2}{|c|}{0,005} \\
\hline \multicolumn{2}{|c|}{5096} & \multicolumn{2}{|c|}{0,060} \\
\hline \multicolumn{2}{|c|}{9408} & \multicolumn{2}{|c|}{0,080} \\
\hline \multicolumn{2}{|c|}{14700} & \multicolumn{2}{|c|}{0,095} \\
\hline \multicolumn{2}{|c|}{19208} & \multicolumn{2}{|c|}{0,105} \\
\hline \multicolumn{2}{|c|}{23520} & \multicolumn{2}{|c|}{0,115} \\
\hline \multicolumn{2}{|c|}{26460} & \multicolumn{2}{|c|}{0,124} \\
\hline \multicolumn{2}{|c|}{25970} & \multicolumn{2}{|c|}{0,150} \\
\hline \multicolumn{2}{|c|}{24990} & \multicolumn{2}{|c|}{0,170} \\
\hline \multicolumn{2}{|c|}{23226} & \multicolumn{2}{|c|}{0,200} \\
\hline & 070 & & 230 \\
\hline & 110 & & 250 \\
\hline & 248 & & 270 \\
\hline & 170 & & 300 \\
\hline & 190 & & 350 \\
\hline & 210 & & 400 \\
\hline & 132 & & 450 \\
\hline & 250 & & 500 \\
\hline & 564 & & 600 \\
\hline & 486 & & 650 \\
\hline & 04 & & 680 \\
\hline & 10 & & 700 \\
\hline & 14 & & 720 \\
\hline & 22 & & 750 \\
\hline & 30 & & 770 \\
\hline & 42 & & 790 \\
\hline & 58 & & 820 \\
\hline & 78 & & 840 \\
\hline & 02 & & 900 \\
\hline & 24 & & 100 \\
\hline & 40 & & 500 \\
\hline & 46 & & 000 \\
\hline
\end{tabular}

Carga vs. Deformación HRFA 
Influencia de la fibra de acero en el control de la tenacidad del hormigón simple

Vol. 2, núm. 2., (2018)

Cesar Eduardo Chávez Vera; Pedro Napoleón Chara Moreira; María Belén Alarcón Valencia; María Milagros Fois Lugo

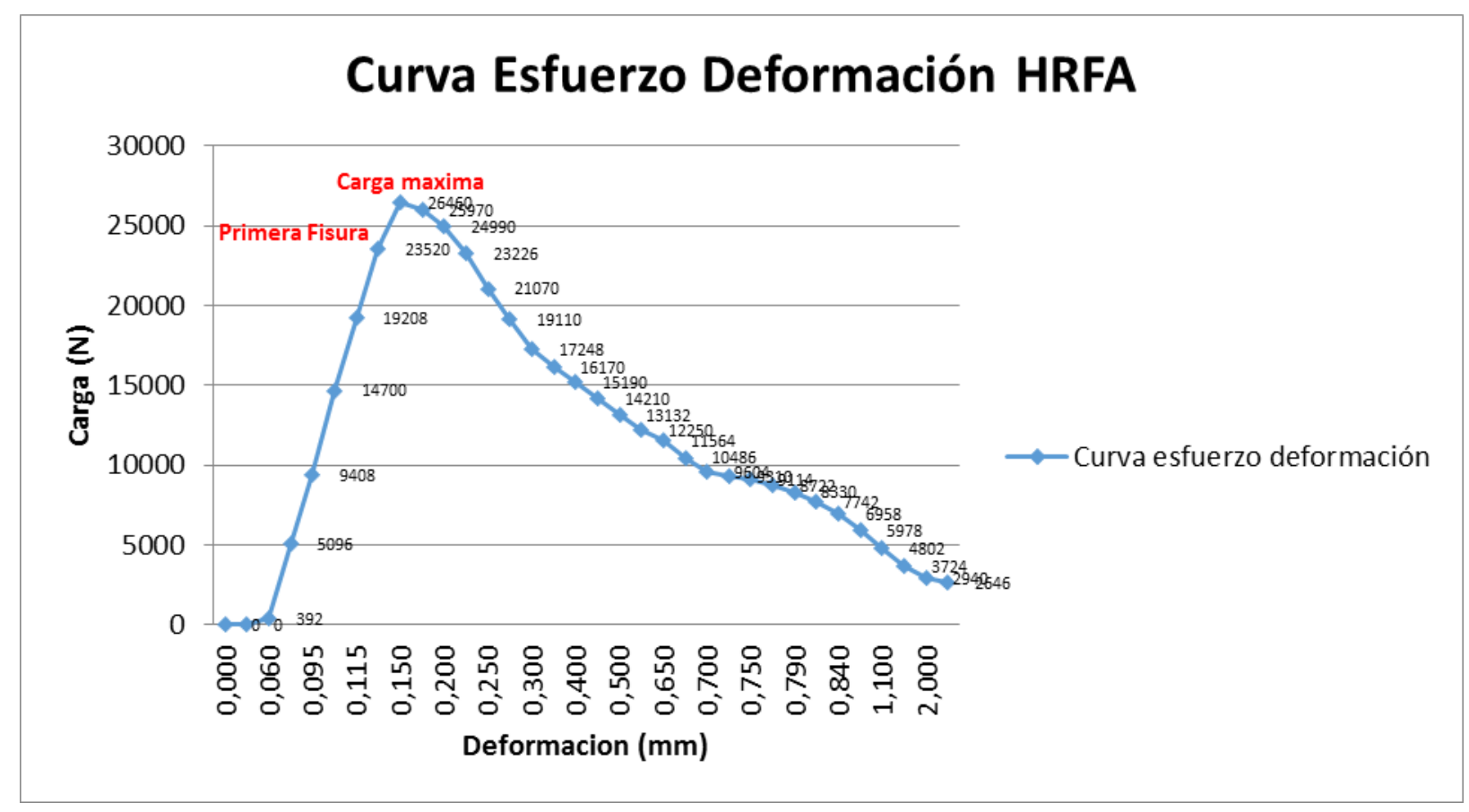

Curva esfuerzo deformación HRFA

Análisis de cargas absorbidas (Tenacidad)

\begin{tabular}{|c|c|c|}
\hline \multicolumn{3}{|c|}{ RESUMEN Y ANÁLISIS DE LA CARGA ABSORBIDA } \\
\hline Nombre de Muestra & Edad de la muestra & $\begin{array}{c}\text { Carga máxima } \\
\text { absorbida (N) }\end{array}$ \\
\hline HS (Hormigón base) & 28 días & $22.148,00$ \\
\hline HRFA & 28 días & $26.460,00$ \\
\hline
\end{tabular}

\begin{tabular}{|c|c|}
\hline $\begin{array}{c}\text { Incremento de Carga } \\
(\mathbf{N})\end{array}$ & $4.312,00$ \\
\hline Incremento $(\boldsymbol{\%})$ & 19,47 \\
\hline
\end{tabular}

\begin{tabular}{|c|c|c|}
\hline \multicolumn{2}{|c|}{ RESUMEN Y ANÁLISIS DE LA DEFORMACIÓN (Flecha) } \\
\hline Nombre de Muestra & Edad de la muestra & $\begin{array}{c}\text { Deformación máxima } \\
(\mathbf{m m})\end{array}$ \\
\hline HS (Hormigón base) & 28 días & 0,085 \\
\hline
\end{tabular}

Revista Científica Mundo de la Investigación y el Conocimiento. 2 (2). pp. 209-235 


\section{Influencia de la fibra de acero en el control de la tenacidad del hormigón simple}

Vol. 2, núm. 2., (2018) Cesar Eduardo Chávez Vera; Pedro Napoleón Chara Moreira; María Belén Alarcón Valencia; María Milagros Fois Lugo

\begin{tabular}{|c|c|c|}
\hline HRFA & 28 días & 2,000 \\
\hline
\end{tabular}

\begin{tabular}{|c|c|}
\hline $\begin{array}{c}\text { Incremento de Carga } \\
(\mathbf{N})\end{array}$ & 1,92 \\
\hline Incremento (\%) & $2.252,94$ \\
\hline
\end{tabular}

Análisis económico del HRFA en comparación al HS

\begin{tabular}{|c|c|c|c|c|}
\hline \multicolumn{5}{|c|}{ COSTO MATERIALES 1 M3 HS } \\
\hline MATERIAL & CANTIDAD & UNIDAD & $\begin{array}{c}\text { COSTO } \\
\text { U. }\end{array}$ & SUBTOTAL \\
\hline CEMENTO & 7,380 & Saco & 7,95 & 58,67 \\
\hline AGUA: & 0,221 & $\mathrm{~m}^{3}$ & 5,00 & 1,11 \\
\hline PIEDRA: & 0,385 & $\mathrm{~m}^{3}$ & 18,57 & 7,15 \\
\hline ARENA: & 0,257 & $\mathrm{~m}^{3}$ & 20,00 & 5,14 \\
\hline \multicolumn{7}{|r}{} & TOTAL: & $\mathbf{7 2 , 0 7}$ \\
\hline
\end{tabular}

\begin{tabular}{|c|c|c|c|c|}
\hline \multicolumn{5}{|c|}{ COSTO MATERIALES 1 M3 HS } \\
\hline MATERIAL & CANTIDAD & UNIDAD & $\begin{array}{c}\text { COSTO } \\
\text { U. }\end{array}$ & SUBTOTAL \\
\hline CEMENTO & 7,380 & Saco & 7,95 & 58,67 \\
\hline AGUA: & 0,221 & $\mathrm{~m}^{3}$ & 5,00 & 1,11 \\
\hline PIEDRA: & 0,385 & $\mathrm{~m}^{3}$ & 18,57 & 7,15 \\
\hline ARENA: & 0,257 & $\mathrm{~m}^{3}$ & 20,00 & 5,14 \\
\hline DRAMIX: & 44,085 & $\mathrm{Kg}$. & 2,80 & 123,44 \\
\hline \multicolumn{7}{r}{} & & TOTAL: & $\mathbf{1 9 5 , 5 1}$ \\
\cline { 4 - 5 }
\end{tabular}

RESUMEN COMPARATIVO

\begin{tabular}{|c|c|c|c|c|c|}
\hline \multirow{2}{*}{ HORMIGÓN } & UNIDAD & CANTIDAD & $\begin{array}{c}\text { COSTO } \\
\text { U. }\end{array}$ & SUBTOTAL & $\%$ \\
\hline HS & M3 & 1 & 72,07 & 72,07 & 100 \\
\hline HRFA & M3 & 1 & 195,51 & 195,51 & 271,280514 \\
\hline
\end{tabular}

\section{Conclusiones.}




\section{Influencia de la fibra de acero en el control de la tenacidad del hormigón simple}

Vol. 2, núm. 2., (2018)

Cesar Eduardo Chávez Vera; Pedro Napoleón Chara Moreira; María Belén Alarcón Valencia; María Milagros Fois Lugo

El incremento de la resistencia a esfuerzos de corte en el hormigón es viable mediante la incorporación de fibra de acero en el diseño de hormigones utilizando la metodología de la ACI.

La trabajabilidad del HRFA respecto del hormigón base, decae en un 15\% debido a la incorporación de fibras de acero, esto determina que para obtener hormigones de alta resistencia es necesario trabajar con revenimientos altos que garanticen la fluidez de la pasta de hormigón, así como medir el uso de la fibra que afecta a su compactación y posterior mente a la resistencia del elemento.

La fabricación de muestras mediante métodos manuales favorece la segregación de las fibras de acero causando un efecto negativo en la resistencia, se recomienda utilizar métodos mecánicos de mezclado como el uso de concretera para que las fibras que por defecto vienen pegadas, se dispersen de manera homogénea en la matriz de hormigón

La resistencia a la compresión de hormigón no dio los resultados esperados, a pesar trabajar con contenidos bajos de fibras se modificó negativamente esta propiedad, por lo que se recomienda experimentar con diferente esbeltez de fibras así como trabajar con criterios de hormigón auto compactante para mantener o mejorar esta propiedad.

El módulo de rotura se incrementó favorablemente mediante la inclusión de fibra de acero, desde este enfoque, se caracteriza la tenacidad del hormigón, la cual mejora notablemente en comparación con el hormigón base además de permitir una mayor deformación de los elementos de hormigón retrasando el colapso o falla total de los mismos. 


\section{Influencia de la fibra de acero en el control de la tenacidad del hormigón simple}

Vol. 2, núm. 2., (2018)

Cesar Eduardo Chávez Vera; Pedro Napoleón Chara Moreira; María Belén Alarcón Valencia; María Milagros Fois Lugo

La determinación de la primera fisura mediante los procedimientos dictados en las normas UNE no son objetivos ya que sugieren la aparición de una primera fisura antes de que el elemento llegue a su carga máxima, esta primera fisura es difícil de determinar a simple vista. Por este motivo para la determinación de la flecha máxima para los ensayos se trabajó con la deformación causada por la carga máxima.

Finalmente, se determina que para un incremento del $19 \%$ de resistencia a cargas de esfuerzos cortantes y $2200 \%$ en capacidad de deformación, el HRFA tiene un costo superior en relación al hormigón base de aproximadamente un 171\%.

\section{Bibliografía.}

American Society for Testing Materials. (1997). ASTM C1018-97, Standard Test Method for Flexural Toughness and First-Crack Strength of Fiber-Reinforced Concrete (Using Beam With Third-Point Loading) (Withdrawn 2006). West Conshohocken, Pensilvania.

GUETTU, R., L., A., AGUADO, A., \& SALDIVAR, H. (2000). La tenacidad de los hormigones reforzados con fibras de acero. Barcelona: Centro internacional de metodos numéricos en Ingenieria, UPC.

Instituto Nacional de Estadisticas y Censos. (2014). Anuario de Estadisticas de Edificaciones. Guayaquil: Dirección de Comunicacion Social INEC.

Instituto Nacional de Estadisticas y Censos. (2015). Anuario de Estadisticas de Edificaciones. Obtenido de Instituto Nacional de Estadisticas y Censos: http://www.ecuadorencifras.gob.ec/encuesta-anual-de-edificaciones-permisos-deconstruccion/

Terreros de Varela, C. (2006). Materiales de Construcción. Guayaquil, Guayas, Ecuador: Centro de difusión y publicaciones ESPOL. 